\title{
COMMENTARY
}

\section{Further observations on enablement}

We welcome the excellent paper by Mead and colleagues on the characteristics and utility of the concept of enablement. ${ }^{1}$ Their paper raises two themes, one on the meaning of enablement, and the second on whether its use as a quality measure would be practical and fair.

The meaning of enablement. The most interesting issue in the first theme arises from the observation that patients from ethnic minority groups report higher enablement while possibly receiving care that might otherwise be expected to produce the opposite effect (in particular, having shorter consultations). In our papers on the same subject, ${ }^{2,3}$ we not only found that ethnic minority groups reported higher enablement, but also that the difference was even greater when the consultation took place in the patient's own language. ${ }^{3}$ This is compatible with Mead et al's suggestion that ethnic minority patients are more likely to have their possibly different expectations of health care met than are native UK patients, ${ }^{1}$ but also suggests that these needs are most likely to be met when the cultural context of doctor and patient are well matched. But the situation may well be even more complex. In a small student study in west London, Hindi and Urdu speaking patients appeared to have different understandings of individual items in the patient enablement instrument (PEI), and also showed different levels of respect towards their GPs (S Khan and GK Freeman, unpublished data, 2004).

We completely agree with Mead et al that more qualitative work is needed to help understand the meaning of enablement for different groups and cultures. In the meantime, however, there is a clear message that practices with significant numbers of ethnic minority patients (often small and apparently disadvantaged practices) are offering their patients something that they rate highly, and that care must be taken to ensure that this is not lost in any reformatting of primary care services, in particular in inner city areas.

The influence of case-mix. The second theme has significant relevance to the incentivising of improved quality of care - mainly through the Quality and Outcomes Framework (QOF). It is generally accepted that the QOF lacks a good measure of the key elements of good interpersonal care, and the possibility of using enablement as a contributor to one is now a reality. Mead et al raise a number of questions about how fair this might be given the associations between enablement and age (higher in the elderly), deprivation (lower), and chronic illness (lower).

Differential effects on enablement in consultations in areas of high and low deprivation have been reported recently in patients with psychological problems. ${ }^{4}$ However, in our own research in four contrasting areas of the UK, ${ }^{2}$ we found that after 50 consecutive consultations case-mix could largely be discounted as a source of variation between doctors' enablement scores. Case-mix statistics for the top and bottom sextiles of enablement scores at doctor level (171 doctors) showed $50.3 \%$ and $50.7 \%$ of patients respectively reporting a mixture of social and psychological problems, and $41.4 \%$ and $43.8 \%$ respectively reporting chronic illness (JGR Howie et al, unpublished data, 2008). The overall rank correlation between doctors' enablement scores and their practices' underprivileged area (UPA) scores was $r=0.14$, between enablement and the percentage of their patients with psychosocial problems was $r=0.03$, and between enablement and the percentage of their patients with chronic illness was $r=-0.14$. On the other hand, where doctors had at least 25 'other language' enablement scores to compare with their 'English language' scores, the correlation was $r=0.72 .^{5}$

So, although we agree again with Mead et al that caution has to be applied before using enablement on its own as an element of the national quality programme, we do not see why it could not contribute to a new QOF 'interpersonal care' measure now. Indeed we have proposed one such measure in the BJGP - the CQI-2. ${ }^{6}$ The CQI-2 uses a combination of enablement (an outcome measure), a proxy for continuity of care, and the CARE measure (a measure of the process of the consultation) and is now being widely used in the assessment of GPs in training. ${ }^{7}$ Although consultation length has been shown to be an important correlate with enablement, ${ }^{2,8,9}$ we have discounted the use of consultation length as a fourth component for the time being, partly because consultation length statistics are not available for consultations under the conditions prevailing since the introduction of the 2004 contract, and partly because consultation length could be open to gaming by responding doctors. The remaining three elements of CQI-2 can be administered without any involvement of the doctor and are a truly independent way of capturing patients' views of important aspects of their consulting experience. Doctors who score highly on these three elements of interpersonal care are often the same (enablement and continuity $r=0.22$; enablement and empathy $r=0.65$ ). ${ }^{6}$

CQI scores are modestly case-mix sensitive being positively related to the proportion of patients over 65 years of age $(r=0.35)$ and to the ratio of chronic to acute patients $(r=0.33)$ : both due to associations with greater continuity of care. In practical terms, this translates into a doctor in the top CQI band as against the lowest CQI band seeing one more patient with a chronic complaint at an average surgery. The correlation with UPA score was not significant at $r=0.06 .^{5}$ 
Other points. As well as using a three-item version of our six-item PEI, Mead et al also used a slightly different approach to scoring enablement to the one we have used in our more recent work. Mead et al use a percentage score, which we did also originally, whereas we now recommend using a mean score as we have found stronger correlations with other variables that way. ${ }^{8}$

We have also researched the impact of 'not applicable' scores on total enablement scores in earlier work, deciding to score 'not applicable' responses as zero as our interest was in measuring the positive outcomes felt by patients. We have accepted questionnaires with up to two 'not applicable' responses for analysis, but exclude responses with more than two.

We note that Mead et al express concern about including enablement in a national incentive programme as there may be inequalities in scores due to the ethnic mix of patients. We have drawn attention to this issue in general terms previously, raising the possibility/probability that similar ethnicity-linked inequalities could apply to other biomedical and administrative process measures in the QOF. For example, there are likely to be ethnic differences in how population groups respond to invitations for health screening, to taking medication for 'silent' risks like raised cholesterol and blood pressure, and to attending for diabetic follow-up. These are more rather than less likely to prejudice against practices with large ethnic minority populations, making the QOF inherently unfair to such practices. There is a strong case for suggesting that the QOF ought to be more widely tested for such possible unfairness. Mead et al must now have the data available to examine this issue, and we hope that they will be able to do this in the near future.

One thing that we are all likely to agree about is the importance of empathic interpersonal skills in helping patients understand and manage their health problems. It is time that healthcare professionals, who value holistic care and work hard to deliver such care to their patients, are recognised and rewarded appropriately for doing so.

\section{John GR Howie,}

Emeritus Professor of General Practice, University of Edinburgh, 4 Ravelrig Park, Balerno, Edinburgh EH14 7DL. Email: john.howie00@btinternet.com

David J Heaney,

Associate Director, Centre for Rural Health, University of Aberdeen, Inverness.

Margaret Maxwell,

Reader, Department of Applied Social Science, University of Stirling, Stirling.

George K Freeman,

Visiting Professor of General Practice, Division of Community Health Sciences, St George's, University of London, London.

Stewart W Mercer,

Professor of Primary Care Research, General Practice \& Primary Care, Division of Community-based Sciences, University of Glasgow, Glasgow.

\section{REFERENCES}

1. Mead N, Bower P, Roland M. Factors associated with enablement in general practice: cross-sectional study using routinely-collected data. Br J Gen Pract 2008; 58: $346-352$. DOI: $10.3399 /$ bjgp08X280218

2. Howie JGR, Heaney DJ, Maxwell M, et al. Quality at general practice consultations: cross sectional survey. BMJ 1999; 319(7212): 738-743.

3. Freeman GK, Walker JJ, Howie JGR, et al. Non-English speakers consulting with the GP in their own language: a cross-sectional survey. Br J Gen Pract 2002; 52: 36-38.

4. Mercer SW, Watt GMC. The inverse care law: clinical primary care encounters in deprived and affluent areas of Scotland. Ann Fam Med 2007; 5: 503-510.

5. Howie JGR, Heaney DJ, Maxwell M, et al. Developing a 'consultation quality index' (CQI) for use in general practice. Fam Pract 2000; 17: 455-461.

6. Mercer SW, Howie JGR. CQI-2 - a new measure of holistic interpersonal care in primary care consultations. Br J Gen Pract 2006; 56: 262-268.

7. Murphy DJ, Bruce DA, Mercer SW, Eva KW. The reliability of workplace-based assessment in postgraduate medical education and training: a national evaluation in general practice in the United Kingdom. Adv Health Sci Educ Theory Pract 2008: DOI 10.1007/s10459-008-9104-8.

8. Howie JGR, Heaney DJ, Maxwell M. Measuring quality in general practice. Occasional Paper 75. London: Royal College of General Practitioners, 1997.

9. Mercer SW, Fitzpatrick B, Gourlay G, et al. More time for complex consultations in a high deprivation practice is associated with increased patient enablement. Br J Gen Pract 2007; 57: 960-966. 